\title{
Scattering of thermal He beams by crossed atomic and molecular beams. III. Anisotropic intermolecular potentials for $\mathrm{He}+\mathrm{N}_{2}, \mathrm{O}_{2}, \mathrm{CO}$, and $\mathrm{NO}^{\mathrm{a})}$
}

\author{
Mark Keil, b) John T. Slankas, and Aron Kuppermann \\ Arthur Amos Noyes Laboratory of Chemical Physics, ${ }^{c)}$ California Institute of Technology, Pasadena, \\ California 91125 \\ (Received 2 May 1978)

\begin{abstract}
Differential scattering cross sections are measured for $\mathrm{He}+\mathrm{N}_{2}, \mathrm{O}_{2}, \mathrm{CO}$, and NO, using the crossed molecular beams technique. These data, which are sensitive to the van der Waals attractive minima and adjacent regions of the intermolecular potentials, are analyzed in terms of both central-field and anisotropic models. Little evidence is found for quenching of the observed diffraction oscillations, and anisotropic contributions are determined to be small: the spherical averages of these anisotropic potentials are indistinguishable, within experimental error, from the potentials obtained by a central-field analysis. This study thus provides a quantitative, empirical validation of the central-field assumption for molecular
\end{abstract} \\ scattering in weakly anisotropic systems.
}

\section{INTRODUCTION}

van der Waals interaction potentials, which are responsible for a wide variety of gas-phase, ${ }^{1,2}$ as well as condensed-phase properties, ${ }^{2,3}$ have been rather well characterized for rare gas atom-atom pairwise interactions. These potentials, determined by a combination of experimental ${ }^{4}$ and theoretical $1^{5}$ techniques, may now be used to correlate experimental data of many different kinds. ${ }^{6-8}$ This success in determining such a detailed microscopic property as the weak van der Waals interatomic potential has been due to a concerted and sustained effort to obtain such information over the past several years.

Many of the techniques so successfully applied to the determination of atom-atom potentials have also been directed towards atom-molecule and molecule-molecule systems. ${ }^{9}$ Because of the additional degrees of freedom, it may be possible to gain more information from studies of van der Waals interactions in molecular systems than in atomic ones: the potentials depend not only on the intermolecular separation, but also on the molecular orientation and intramolecular configuration. ${ }^{10,11}$ Very often however, the internal structure of the molecular partner(s) is neglected; a central-field analysis is then used to determine what is hoped to be the spherically symmetric component of the full (anisotropic) potential. ${ }^{9}$ This central-field analysis has until recently ${ }^{10,11}$ been necessitated by the complexities inherent in analyzing anisotropic interactions. The validity of the centralfield analyses are thus predicated upon a combination of orientational averaging of the potentials, which would result in "effective" spherically symmetric potentials, and upon negligibly small anisotropies in the intermolecular potentials of the systems considered.

\footnotetext{
a) This work was supported in part by a Contract (EY-76-S-03767) from the Department of Energy. Report Code: CALT767P4-174.

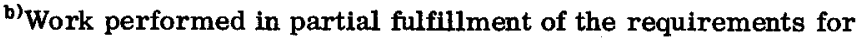
the Ph.D. degree in Chemistry at the California Institute of Technology.

c) Contribution No. 5776 .
}

Anisotropic effects for molecular scattering have been investigated both experimentally and theoretically. Quenching of the oscillatory structure both for semiclassical and quantal systems has been predicted, ${ }^{12-14}$ and has been verified experimentally. ${ }^{11,15}$ However, with most systems studied to date, the observed effects have been rather small. ${ }^{15}$ In such cases, the lack of strong quenching has been taken as indicative of small anisotropies, and the central-field analysis has been applied, usually without any further justification. ${ }^{\theta}$ Recently how ever, it has become clear that anisotropic effects for the scattering of atoms by highly nonspherical molecules yield central-field potentials which are quite different from the spherically symmetric components of the actual anisotropic potentials. ${ }^{11}$ Consequently, the validity of central-field analyses of scattering in less highly anisotropic systems must be verified quantitatively. Phrasing the problem somewhat differently, we need to know just how nearly isotropic the system must be in order to validate the central-field analysis. It is only when the central-field analysis is incapable of fitting the data that information about the anisotropy of the potential can be deduced from measurements that do not distinguish between elastic and concurrent inelastic processes.

In the present paper, we analyze experimental measurements of the differential cross sections (DCS) for the highly quantum systems $\mathrm{He}+\mathrm{N}_{2}, \mathrm{O}_{2}, \mathrm{CO}$, and $\mathrm{NO}$, obtained using room-temperature crossed beams. These systems were chosen as representative of nonhydride molecules which should be weakly anisotropic. This is expected on the basis that the diatomic bond lengths are significantly smaller (by a factor of $\sim 2.5$ ) than the smallest intermolecular separations to which the present DCS data are sensitive. In Sec. II, we briefly describe the apparatus used to measure the DCS's and the data reduction procedures used to interpret them. The DCS measurements, and the central-field and anisotropic intermolecular potentials extracted from them, are presented in Sec. III. In Sec. IV, we discuss the appropriateness of the central-field analysis for these systems and compare our potentials to those obtained from other experiments. Systematic trends in the po- 
TABLE I. Beam operating conditions.

\begin{tabular}{|c|c|c|c|c|c|}
\hline \multirow{2}{*}{$\frac{\text { Characteristic }}{\text { Beam gas }}$} & \multicolumn{2}{|l|}{ Primary beam } & \multicolumn{3}{|c|}{ Secondary beams } \\
\hline & $\mathrm{He}$ & $\mathrm{N}_{2}$ & $\mathrm{O}_{2}$ & $\mathrm{CO}$ & NO \\
\hline Inlet pressure/torr & 1300 & 3.8 & 3.8 & 3.8 & 4.9 \\
\hline Inlet temperature $/ \mathbf{K}$ & 298 & 298 & 298 & 298 & 298 \\
\hline Angular FWH $\mathrm{M}^{\mathrm{a}} / \mathrm{deg}$ & 1 & 4 & 4 & 4 & 5 \\
\hline Most probable velocity $/(\mathrm{km} / \mathrm{sec})$ & 1.757 & 0.599 & 0.561 & 0.599 & 0.579 \\
\hline Velocity $\mathrm{FWHM}^{\mathrm{a}}{ }^{\mathrm{a}} \Delta v / v$ & 0.10 & 0.75 & 0.75 & 0.75 & 0.75 \\
\hline Mach number ${ }^{16}$ & 18 & 1.5 & 1.5 & 1.5 & 1.5 \\
\hline
\end{tabular}

afull width at half-maximum.

tentials for the four systems studied herein are also discussed. Finally, we summarize our findings in Sec. V.

\section{EXPERIMENTAL AND DATA REDUCTION}

The crossed molecular beam apparatus used in this study has been described previously, ${ }^{8}$ and is discussed below only very succinctly. The modulated He primary beam is formed in a supersonic expansion, and is angularly well collimated in two stages of differential pumping. Secondary beams of the various gases studied emerge from a glass capillary array, directly into the scattering chamber, and are intense but only slightly supersonic. The secondary beam source may be tilted upwards, thus uncrossing the beams, to allow measurements of the modulated primary beam scattered by background gas. Beam operating characteristics are summarized in Table $I$. Due to the slightly supersonic nature of the secondary beam, its internal temperature (particularly its rotational temperature) may not be the ambient one $(298 \mathrm{~K})$. We note that its velocity distribution is best described by a Mach number of 1.5 (Table I) as well as an effective specific heat ratio of $1.64,{ }^{16}$ the latter indicating only a very slight cooling of internal states.

A quadrupole mass spectrometer detector having an electron bombardment ion source may be positioned at a variable angle in, or out of, the plane of the beams. These experiments were performed with out-of-plane detection (fixing the in-plane angle at $0^{\circ}$ ) of scattered He atoms. This selection of scattering geometry increases the laboratory angular spacings between the observed diffraction oscillations, enabling a better characterization. ${ }^{17}$ In addition, experiments with $\mathrm{He}+\mathrm{Ar}$ using this geometry yielded potentials whose fits to the DCS were of consistently higher quality than were those of the in-plane detection geometry. ${ }^{B}$ Ion detection and signal processing in the se experiments utilized a 14stage $\mathrm{Cu}-\mathrm{Be}$ dynode electron multiplier, followed by several stages of analogue preamplification, and a lockin amplifier whose reference signal input was synchronized with the primary beam chopper. The output signal was digitized for sampling and averaging by a laboratory minicomputer, which also performed background signal subtraction by uncrossing the beams and remeasuring the modulated signal.

Differential pumping in the mass spectrometer is provided by a small $(5 \mathrm{l} / \mathrm{s})$ ion pump in the detector's entrance buffer chamber, followed by a larger $(25 \mathrm{l} / \mathrm{s})$ ion pump in the ionizer region. The latter is supplemented during experiments by a liquid helium cryopump ( 400 $1 / \mathrm{s}$ ). In a very clean environment, such as provided by baking out the detector at $\sim 200^{\circ} \mathrm{C}$ for $\sim 20 \mathrm{~h}$, the electropolished stainless steel surface of the liquid helium dewar provides an effective pump for all gases, including $\mathrm{Ne}, \mathrm{H}_{2}$, and even $\mathrm{He} !^{8}$ The pressure in the ion source region was about $5 \times 10^{-10}$ torr during the course of these experiments.

Maintaining the scattering chamber pressure below $3 \times 10^{-8}$ tor $r$, and keeping the primary beam attenuation due to scattering by the secondary beam at $\sim 5 \%$, ensures that the single-collision conditions essential for DCS measurements prevail. The slight cryopumping of NO on liquid-nitrogen cooled surfaces in the scattering chamber allowed use of a somewhat higher secondary beam pressure for this gas which, however, increased this beam's angular divergence slightly (see Table I). Periodic mass spectrometric checks of the secondary beams revealed their high purity for all the scattering experiments, with no discernible dimerization.

Scattered primary beam signals were measured between $\sim 2.7^{\circ}$ and $\sim 16.5^{\circ}$ above the plane of the beams, at a relative collision energy of $\sim 64 \mathrm{meV}$ (the exact values depend on the identity of the secondary beam gas). Measurements were also made for out-of-plane scattering below the plane of the beams, in order to locate accurately the zero position of the angular scattering scale (to within $0.03^{\circ}$ ). ${ }^{8}$ Long-term drifts in beam intensities and detection efficiency were compensated for by periodic measurements of the scattering signal at a reference angle near $5^{\circ}$. The experimental DCS's are shown in Fig. 1 for $\mathrm{He}+\mathrm{N}_{2}, \mathrm{He}+\mathrm{O}_{2}, \mathrm{He}+\mathrm{CO}$, and $\mathrm{He}+\mathrm{NO} .^{18}$ Data points represent the average of two or three independent angular scans performed in several days' experimentation for each scattering pair; total integration times at each angle ranged from $\sim 2$ min near $3^{\circ}$, to $\sim 30 \mathrm{~min}$ at the widest angles. Signal-to-noise ratios over the same angular range were between about 40 and 6 , respectively.

The data reduction procedure used to obtain the fits shown in Fig. 1 has been described previously. ${ }^{8}$ Using Marquardt's weighted least-squares prescription, ${ }^{19}$ we fit the parameters of mathematical functions chosen to represent the intermolecular potential, as discussed in Sec. III. DCS's in the center-of-mass coordinate frame are calculated from partial wave phase shifts of high ac- 


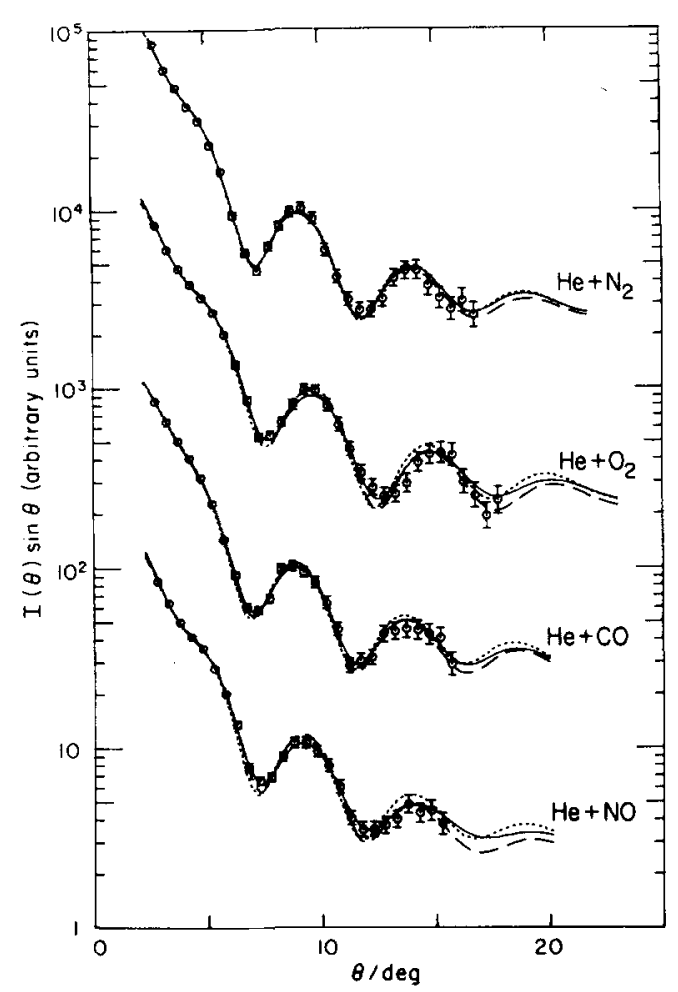

FIG. 1. Laboratory differential cross sections for out-of-plane scattering of $\mathrm{He}$ by $\mathrm{N}_{2}, \mathrm{O}_{2}, \mathrm{CO}$, and $\mathrm{NO}$, with ordinates shifted downwards by one decade for each system in this sequence. Points with error bars are the experimental measurements of the scattering cross sections ${ }^{18} I(\theta) \sin ^{\theta}$ vs the scattering angle $\theta$ at a relative collision energy of $64 \mathrm{meV}$. Curves are DCS's calculated using potentials extracted from the data. Solid lines: anisotropic MSV potential; dashed lines: central-field SPFD potential; dotted lines: spherical average of the anisotropic MSV potential. The potential models are presented in Eqs. (3)-(6); their parameters are given in Tables II and III.

curacy, and are averaged extensively over the angular and velocity spreads of both beams at the time the transformation to the out-of-plane laboratory coordinate frame is performed. The overall apparatus velocity resolution is $\sim 12 \%$ FWHM for the systems studied here. Additional angular averaging is also used to account for the overall apparatus angular resolution, of $1.9^{\circ}$ FWHM. $^{8}$

The functional to be minimized in the least-squares fit is given by

$$
\chi^{2}=\sum_{i=1}^{n} g_{i}\left(I_{i}-\alpha \sigma_{i}\right)^{2}
$$

Following Paper II, we define a goodness-of-fit statistical index more suitable than $\chi^{2}$ for describing the quality of the fits to the DCS,

$$
\frac{\Delta \alpha_{0.05}}{\alpha}=\frac{1}{\alpha} t_{0.05}(n-k)\left[\frac{\chi^{2}}{(n-k) \sum_{i=1}^{n} g_{i} \sigma_{i}^{2}}\right]^{1 / 2} \text {. }
$$

In these equations, $I_{i}$ and $\sigma_{i}$ are the measured (arbitrarily normalized) and calculated (absolute) cross sections ${ }^{18}$ at each of $n$ scattering angles, respectively, with the normalized weights $g_{i}$. For $n$ data points and $k$ parameters, $t_{0.05}(n-k)$ is the appropriate Student $t$-distribution statistic for a $95 \%$ confidence level of the scaling factor $\alpha{ }^{20}$ Note that the $\Delta \alpha_{0.85} / \alpha$ statistical index is independent of the arbitrary normalization used in the experiment, and furthermore is statistically adjusted for the number of data points. This should allow a more straightforward comparison between the results from different laboratories.

\section{DATA ANALYSIS AND INTERMOLECULAR POTENTIALS}

The experimental DCS's obtained as described in Sec. II and displayed in Fig. 1 were fitted by weighted least squares optimization of the parameters of the intermolecular potentials described below. These potentials are written in the reduced form

$$
f(\rho)=\frac{V(r)}{\epsilon}, \rho=\frac{r}{r_{m}},
$$

where $r_{m}$ is the radius of the attractive minimum and $\epsilon$ is its depth. The zero of the potential, occurring at $r=\sigma$, is related to $r_{m}$ and the potential shape $f(\rho)$, and is not an independent parameter.

The mathematical shape functions $f(\rho)$ used in the present study include the familiar LJ12-6 potential form,$^{2}$ in order to allow a comparison with results from measurements of integral scattering cross sections. ${ }^{21,22}$ More flexible potential shape functions are however, required to accurately describe the weak van der Waals interactions analyzed here ${ }^{8,23,24}$ One such potential function $^{23}$ is the Morse-spline-van der Waals (MSV) parameterization, ${ }^{25}$ given by

$$
\left.\begin{array}{lc}
f(\rho)=e^{\Delta(1-\rho)}\left[e^{\beta(1-\rho)}-2\right] & \text { for } \rho=\rho_{1} \\
f(\rho)=\left(\rho_{2}-\rho\right)\left[s_{1}\left(\rho_{2}-\rho\right)^{2}+s_{3}\right]+\left(\rho-\rho_{1}\right)[ & \left.s_{2}\left(\rho-\rho_{1}\right)^{2}+s_{4}\right] \\
f(\rho)=-\sum_{i=1}^{3} c_{2 i+4} \rho^{-(2 t+4)} & \text { for } \rho_{1}<\rho<\rho_{2}
\end{array}\right\}
$$

The cubic spline coefficients $s_{i}(i=1$ through 4) are fixed by smoothness conditions at the spline points $\rho_{1}$ and $\rho_{2}$, with the first one being chosen as the inflection point of the Morse function, ${ }^{23} \rho_{1}=1+\beta^{-1} \ln 2$. The second spline point is fixed at $\rho_{2}=1.6$. $^{8}$

It has recently been shown that room-temperature DCS measurements of the type considered here enable up to five independent potential parameters to be determined, providing that the parameterization in which they are used has the appropriate flexibility. ${ }^{23}$ In particular, the MSV form of Eq. (4) necessarily couples the weakly repulsive wall region of the potential with the attractive minimum, since the Morse $\beta$ parameter is used to describe both these regions simultaneously. The present room-temperature data, in addition to being sensitive to the attractive well region of the potential, are also somewhat sensitive to the weakly repulsive wall (down to $\rho \simeq 0.8$ ) and to the long-range attraction (up to $\rho \simeq 1.7$ ), and the coupling between these two regions inherent in the MSV form tends to yield attractive minima which are systematically too deep. ${ }^{8,23}$ Consequently, we also analyzed the data using a modified MSV potential, which has a second Morse function joined to the first at $r=\sigma .^{26}$ 
This $\mathrm{M}^{2} \mathrm{SV}$ form uses the $\beta^{\prime}$ parameter to describe the potential for $r<\sigma$, but is otherwise identical to the MSV potential of $\mathrm{Eq}$. (4). Another parameterization that successfully decouples the attractive minimum region from adjacent regions of the potential is the third-order Simons-Parr-Finlan Dunham (SPFD) potential, ${ }^{27}$ given by

$$
\left.\begin{array}{l}
f(\rho)=b_{0} \lambda^{2}\left(1+\sum_{i=1}^{3} b_{i} \lambda^{i}\right)-1 \text { for } \lambda=1-\frac{1}{\rho} \text { and } \rho<\rho_{f} \\
f(\rho)=-\sum_{i=1}^{3} c_{2 i+4} \rho^{-(2 i+4)} \quad \text { for } \rho \geqslant \rho_{f} .
\end{array}\right\}
$$

The two highest-order coefficients of the SPFD expansion $\left(b_{2}\right.$ and $\left.b_{3}\right)$ are fixed by smoothness conditions ${ }^{28}$ at $\rho_{f}=1.6 .^{8}$

For reduced distances below $\rho=0.65$ (to which our DCS data are not sensitive ${ }^{23}$ ), the SPFD potential often shows spurious maxima or oscillations. For numerical convenience in the phase shift calculation, these irregularities are removed by using a Born-Mayer exponentially repulsive wall, i.e., $f(\rho)=A e^{-b \rho}$, from some joining point $\rho_{i}$ downwards. $A$ and $b$ are fixed by smoothness conditions at $\rho=\rho_{i}$; trial calculations show that values of $\rho_{i}<0.75$ have no effect on the scattering results, and we usually use $\rho_{i}=0.70 .^{23}$

Although nonreduced $C_{8}$ dispersion constants (not to be confused with their reduced counterparts $c_{8}$, where $C_{B}=\epsilon r_{m}^{6} c_{6}$ ) have been calculated for all four systems considered here, ${ }^{29-31}$ we felt it best to fit this region of the potential, for reasons discussed below. Thus, by fixing $c_{8}=c_{10}=0$ in Eqs. (4) and (5), we obtain "effective" dispersion constants, termed $C_{6}^{\prime}$ for each interaction pair. These are expected to be somewhat greater than the corresponding actual $C_{6}$ constants, because of our neglect of higher-order dispersion terms, which have not yet been calculated. The $c_{6}$ parameter obtained bears significance only in describing the scattering data, and in describing the region of the potential to which these data are sensitive. This region probably does not extend as far as the $6.5 \AA$ upper limit attained for the He-Ar potentials of Paper II, because the present experiments include low-angle scattering data down to $\sim 2.7^{\circ}$, instead of $1.9^{\circ} .^{8}$ As a further consequence, our accuracy is probably less than $20 \%$ in the "attractive tail" region beyond $\sim 5 \AA . .^{23}$ For these reasons, the $C_{6}$ parameter is optimized in these fits instead of being held fixed at its theoretical value. The MSV potential thus has four adjustable parameters $\left(r_{m}, \epsilon, \beta\right.$, and $\left.c_{\theta}\right)$, while the $\mathrm{M}^{2} \mathrm{SV}$ and SPFD potentials each have five $\left(r_{m}, \epsilon, \beta, \beta^{\prime}\right.$, and $c_{6} ; r_{m}, \epsilon, b_{0}, b_{1}$, and $c_{6}$, respectively).

A prerequisite for $\mathbf{E q} .(3)$, together with the reduced potential forms of Eqs. (4) and (5), to provide an accurate description of the intermolecular interactions of the systems studied here, is that anisotropic contributions to the potential must be small over the intermolecular distance range to which the DCS data are sensitive (2.8 $₹ r \approx 6 \AA) .{ }^{23}$ It is only in such a case that the potential is independent of the molecular orientation. Although this central-field assumption may not be made a priori for the analysis of atom-molecule scattering experi- ments, we nevertheless base a "first-order" extraction of intermolecular potentials on it.

A possible procedure for more appropriately modeling the systems studied here is to incorporate anisotropic contributions to the intermolecular potentials in the $r_{m}$ and $\epsilon$ parameters of Eq. (3), using an elliptical modification to central-field types of potentials ${ }^{11}$ :

$$
\begin{aligned}
& V(r, \gamma)=\epsilon(\gamma) f[\rho(\gamma, \gamma)] \text {, where } \rho(r, \gamma)=\frac{r}{r_{m}(\gamma)} \text {, and } \\
& r_{m}(\gamma)=r_{m_{1}}\left(\frac{1+r_{q} \sin ^{2} \gamma}{1+r_{q}}\right)^{1 / 2} ; \epsilon(\gamma)=\epsilon_{0}+\epsilon_{2} P_{2}(\cos \gamma) \text {. }
\end{aligned}
$$

In this expression $r$ is the length of the position vector $r$ of the He atom with respect to the molecular center of mass, and $\gamma$ is the angle between $r$ and the molecular axis. For arbitrary $\gamma, r_{m}(\gamma)$ is the value of $r$ at the potential minimum, and $-\epsilon(\gamma)$ is the corresponding potential energy. The parameters $r_{a}$ and $\epsilon_{2}$ are measures of the anisotropy of the intermolecular potential. Any central-field reduced potential energy function $f(\rho)$ may be used, since Eq. (6) introduces anisotropy into the potential through the elliptically shaped $r_{m}(\gamma)$ and the Legendre polynomial representation for $\epsilon(\gamma)$. Scattering calculations for the anisotropic potential model of Eq. (6) are performed using the infinite-order sudden approximation, ${ }^{14 a}$ as discussed in Sec. IV. Because the MSV potential form for $f(p)$ is known to provide a reasonably good representation for spherically symmetric interactions without requiring too many adjustable parameters, ${ }^{23}$ we use this form in Eq. (6) for the present anisotropic potential fits.

Since the central-field MSV potential already has four adjustable parameters, we may adjust only one of the anisotropy parameters of Eq. (6) to the data. ${ }^{23}$ We choose to fix the $\epsilon_{2} / \epsilon_{0}$ ratio (see Sec. IV A), while adjusting $r_{q}$ because of its greater influence on the rapid quantum ascillatory structure of the DCS. ${ }^{14 \mathrm{~b}}$ The five adjustable parameters of the anisotropic potential model of Eqs. (4) and (6) are thus $r_{m_{1}}, \epsilon_{0}, r_{q}, \beta$, and $c_{6}$. Unfortunately, the $D_{\infty h}$ symmetry of our "cigar-shaped" model potential of Eq. (6) is inappropriate for the $\mathrm{He}-\mathrm{CO}$ and $\mathrm{He}-\mathrm{NO}$ interactions. A "pear-shaped" model having the appropriate $C_{\infty \nu}$ symmetry would, however, require at least one additional parameter. This further refinement should have a rather small effect; we note for example that the dipole moments of $\mathrm{CO}$ and $\mathrm{NO}$ are $-0.112^{32}$ and $\pm 0.158 \mathrm{D},{ }^{33}$ respectively (the sign has not yet been determined for NO). These dipole moments are too small to produce the large distortions in the overall electronic cloud distribution that presumably would necessitate a model with $C_{\operatorname{mav}}$ symmetry. By comparing the goodness of fit [to the DCS data, as measured by the $\Delta \alpha_{0.95} / \alpha$ statistical index of Eq. (2)] of the anisotropic potential with those of the central-field $\mathrm{M}^{2} \mathrm{SV}$ and SPFD potentials, all of which have five adjustable parameters, it may be possible to gauge the appropriateness of the model potential of Eq. (6).

Potential parameters for the $\mathrm{He}+\mathrm{N}_{2}, \mathrm{O}_{2}, \mathrm{CO}$, and $\mathrm{NO}$ van der Waals interactions are given in Table II for the fits to the central-field MSV, $\mathrm{M}^{2} \mathrm{SV}$, and SPFD potentials of Eqs. (4) and (5). The $\Delta \alpha_{0.95} / \alpha$ goodness-of-fit 
TABLE II. Central-field intermolecular potentials fit to the experimental DCS's.

\begin{tabular}{|c|c|c|c|c|c|c|c|c|}
\hline System $^{\mathrm{a}}$ & Potential type ${ }^{b}$ & $r_{m} / \AA$ & $\sigma / \AA$ & $\epsilon / \mathrm{meV}$ & Shape parameters ${ }^{c}$ & $Q^{\mathrm{d}} / \AA^{2}$ & $x^{2} \theta, 1$ & $\Delta \alpha_{0.95} / \alpha^{f}(\%)$ \\
\hline \multirow[t]{3}{*}{$\mathrm{He} \div \mathrm{N}_{2}(29)$} & MSV & 3.67 & 3.24 & 2.50 & $\beta=5.82 ; c_{6}=2.15$ & 87 & $130(4)$ & 1.74 \\
\hline & $\mathrm{M}^{2} \mathrm{SV}$ & 3.72 & 3.24 & 2.15 & $\beta=5.30 ; \beta^{\prime}=9.09 ; c_{6}=1.88$ & 82 & $100(5)$ & 1.58 \\
\hline & SPFD & 3.65 & 3.22 & 2.27 & $b_{0}=31.9 ; b_{1}=-4.72 ; c_{6}=2.10$ & 83 & $110(5)$ & 1.64 \\
\hline \multirow{3}{*}{$\mathrm{He}+\mathrm{O}_{2}(31)$} & MSV & 3.44 & 3.05 & 3.05 & $\beta=6.03 ; c_{6}=1.95$ & 83 & $200(4)$ & 2.22 \\
\hline & $\mathrm{M}^{2} \mathrm{SV}$ & 3.49 & 3.04 & 2.52 & $\beta=5.35 ; \beta^{\prime}=12.1 ; c_{6}=1.50$ & 76 & $160(5)$ & 2.01 \\
\hline & SPFD & 3.40 & 3.03 & 2.73 & $b_{0}=36.3 ; b_{1}=-5.45 ; c_{6}=1.85$ & 77 & $160(5)$ & 2.02 \\
\hline \multirow[t]{3}{*}{$\mathrm{He}+\mathrm{CO}(27)$} & MSV & 3.73 & 3.32 & 2.43 & $\beta=6.27 ; c_{6}=1.62$ & 84 & $190(4)$ & 1.91 \\
\hline & $\mathrm{M}^{2} \mathrm{SV}$ & 3.77 & 3.32 & 2.09 & $\beta=5.77 ; \beta^{\prime}=10.3 ; c_{6}=1.29$ & 79 & $160(5)$ & 1.78 \\
\hline & SPFD & 3.70 & 3.31 & 2.28 & $b_{0}=39.2 ; b_{1}=-5.10 ; c_{6}=1.52$ & 81 & $160(5)$ & 1.81 \\
\hline \multirow[t]{3}{*}{$\mathrm{He}+\mathrm{NO}(26)$} & MSV & 3.69 & 3.18 & 2.56 & $\beta=5.03 ; c_{6}=1.78$ & 90 & $320(4)$ & 2.55 \\
\hline & $\mathrm{M}^{2} \mathrm{SV}$ & 3.73 & 3.17 & 2.12 & $\beta=4.66 ; \beta^{\prime}=9.33 ; c_{6}=1.15$ & 79 & $240(5)$ & 2.23 \\
\hline & SPFD & 3.67 & 3.16 & 2.16 & $b_{0}=19.1 ; b_{1}=-3.98 ; c_{6}=1.56$ & 82 & $250(5)$ & 2.31 \\
\hline
\end{tabular}

aExperimental conditions given in Table I; the number of experimental data points is given in parentheses.

Defined in Eqs. (3)-(5).

Symbols have the same meanings as in Eqs. (3)-(5) of the text. The MSV and $\mathrm{M}^{2} \mathrm{SV}$ potentials have $\rho_{2}=1.60$; the SPFD potential similarly has $\rho_{f}=1.60$. The dispersion constant $C_{6}^{\prime}$ is obtained from the fitted reduced dispersion coefficient $c_{6}$, where $C_{6}^{\prime}=\epsilon \gamma_{m}^{6} c_{6}$. The prime denotes an "effective" dispersion coefficient, obtained by neglecting higher-order contributions.

Integral cross sections at a relative collision energy of $64 \mathrm{meV}$ calculated from the partial wave expansion and the potentials given.

'Scattering intensity normalized to 500 at $\theta=4.8^{\circ}$; the number of parameters varied is given in parentheses.

fSee Eqs. (1) and (2).

index [Eq. (2)] displayed in this table statistically accounts for the number of fitted parameters..$^{8,20}$ It may immediately be seen that the five-parameter $M^{2} S V$ and SPFD potentials are better able to fit the DCS data than is the MSV model. Although the size parameters $r_{m}$ and $\sigma$ are seen to be largely model independent $( \pm 0.04 \AA \stackrel{m}{\mathrm{~h}}$ for $r_{m}$ and $\pm 0.01 \AA$ for $\sigma$ ), the MSV potentials give attractive minima consistently deeper than those of the $\mathrm{M}^{2} \mathrm{SV}$ and SPFD potentials, by $\sim 15 \%$. The latter two potentials' $\epsilon$ values are however, within $5 \%$ of one another for each interaction pair. Under conditions of validity of the central-field assumption, the $M^{2} S V$ and SPFD parameterizations yield potentials which are accurate, when fitted to room-temperature DCS data for the present highly quantum systems, to about $\pm 10 \%$ in the van der Waals attractive minimum region. ${ }^{23}$ The DCS data are fitted about equally well by either the $M^{2} S V$ and
SPFD potentials, with the quality-of-fit being quite good except possibly for the $\mathrm{He}+\mathrm{NO}$ system. We also display in Table II the integral cross sections $Q$ at a relative collision energy of $64 \mathrm{meV}$, calculated for each system studied using the partial wave expansion. Because of the consistently greater well depths of the MSV potential, their $Q$ are almost $10 \%$ larger than those of the corresponding $\mathrm{M}^{2} \mathrm{SV}$ or SPFD potentials.

In Table III we list the parameters obtained by fitting the anisotropic MSV potential model of Eqs. (4) and (6) to the DCS data. In the last three columns of this table, we also list the position and depth parameters for the spherical averages of these anisotropic potentials; we note that these are in quite close agreement with the corresponding parameters of the central-field $\mathrm{M}^{2} \mathrm{SV}$ and SPFD potentials as listed in Table II. In Figs. 2-5 we

TABLE III. Intermolecular potential parameters from anisotropic fits to the experimental DCS's.

\begin{tabular}{|c|c|c|c|c|c|c|c|c|c|c|c|}
\hline \multirow[b]{2}{*}{ System ${ }^{a}$} & \multicolumn{6}{|c|}{ Anisotropic potential parameters ${ }^{b}$} & \multirow[b]{2}{*}{$\chi^{2 \mathrm{c}, \mathrm{d}}$} & \multirow[b]{2}{*}{$\Delta \alpha_{0.95} / \alpha^{\mathrm{d}}(\%)$} & \multicolumn{3}{|c|}{$\begin{array}{l}\text { Spherically averaged anisotropic } \\
\text { potential parameters }\end{array}$} \\
\hline & $r_{m \perp} / \AA$ & $r_{q}$ & $\epsilon_{0} / \mathrm{meV}$ & $\epsilon_{2} / \mathrm{meV}$ & $\beta$ & $c_{6}$ & & & $r_{m} / \AA$ & $\sigma / \AA$ & $\epsilon / \mathrm{meV}$ \\
\hline $\mathrm{He}+\mathrm{N}_{2}(29)$ & 3.60 & -0.13 & 2.43 & -0.49 & 5.75 & 2.24 & $130(5)$ & 1.77 & 3.69 & 3.25 & 2.39 \\
\hline $\mathrm{He}+\mathrm{O}_{2}(31)$ & 3.29 & -0.24 & 2.94 & -0.59 & 5.90 & 2.13 & $140(5)$ & 1.92 & 3.48 & 3.07 & 2.76 \\
\hline $\mathrm{He}+\mathrm{CO}(27)$ & 3.58 & -0.22 & 2.35 & -0.47 & 6.25 & 1.75 & $120(5)$ & 1.59 & 3.76 & 3.35 & 2.20 \\
\hline $\mathrm{He}+\mathrm{NO}(26)$ & 3.45 & -0.30 & 2.45 & -0.49 & 4.98 & 1.98 & $100(5)$ & 1.46 & 3.73 & 3.21 & 2.27 \\
\hline
\end{tabular}

${ }^{2}$ Experimental conditions given in Table $I$; the number of experimental data points is given in parentheses.

'Potential form defined by Eqs. (4) and (6); symbols have the same meanings as in these equations. The MSV potential of Eq. (4) has $\rho_{2}=1.60$; the $\epsilon_{2} / \epsilon_{0}$ ratio is fixed at -0.2 as discussed in Sec. IV.

"Scattering intensity normalized to 500 at $\theta=4.8^{\circ}$; the number of parameters varied is given in parentheses.

See Eqs. (1) and (2). 


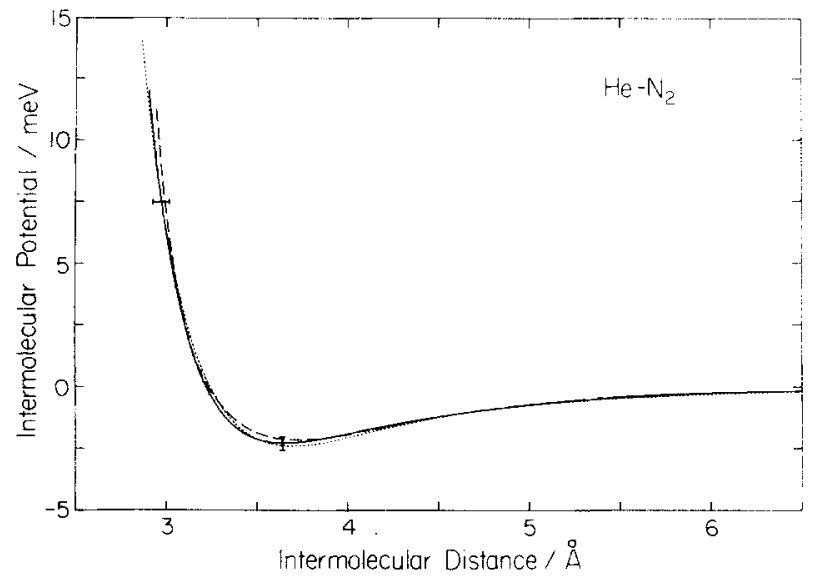

FIG. 2. Spherically symmetric intermolecular potentials for $\mathrm{He}+\mathrm{N}_{2}$ as a function of intermolecular distance. Solid curve: central-field SPFD potential; dashed curve: central-field $\mathrm{M}^{2} \mathrm{SV}$ potential; dotted curve: spherical average of the anisotropic MSV potential. The error bars on the SPFD potential represent the accuracy with which it has been determined, $\pm 10 \%$ of the potential energy at the attractive minimum, $\pm 0.04 \AA$ in the position of the repulsive wall at $\sim 7.5 \mathrm{meV}$.

plot these three spherically symmetric potentials for the $\mathrm{He}+\mathrm{N}_{2}, \mathrm{O}_{2}, \mathrm{CO}$, and NO systems, respectively. For each of the four systems studied, the central-field potentials are seen to be substantially the same as the spherical average of the anisotropic potentials (see also the following discussion).

\section{DISCUSSION}

\section{A. Central-field and anisotropic potential analysis}

The van der Waals interactions for the atom-molecule systems considered here are anisotropic. As such, the central-field potentials generically represented by Eq. (3) provide at best an approximate description of these interactions. Consequently, the first problem to be addressed is the validity of the central-field assumption. In the discussion that follows, we shall present a quantitative assessment of this assumption.

Even if the central-field assumption were invalid with-

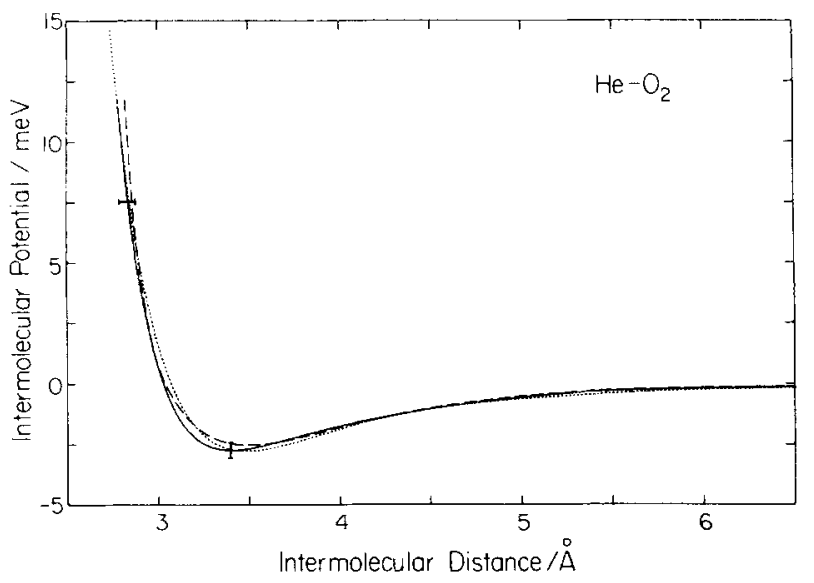

FIG. 3. Spherically symmetric intermolecular potentials for $\mathrm{He}+\mathrm{O}_{2}$; symbols as in Fig. 2 .

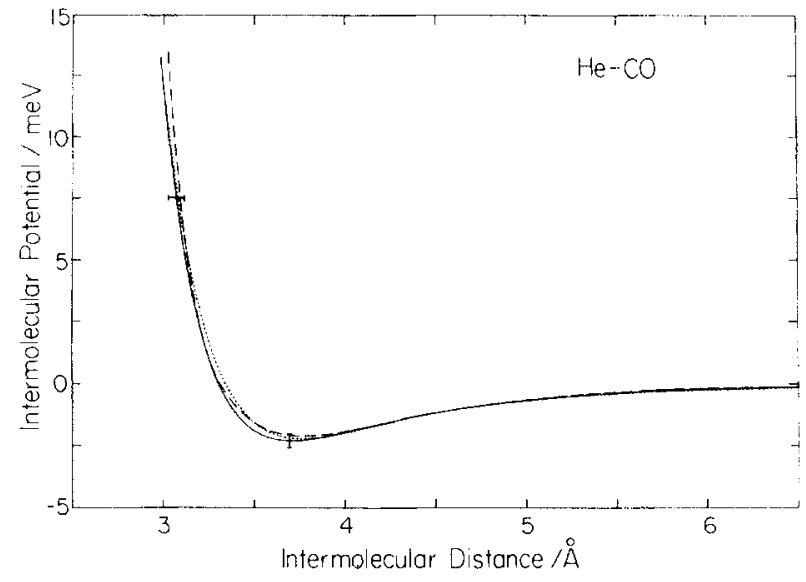

FIG. 4. Spherically symmetric intermolecular potentials for $\mathrm{He}+\mathrm{CO}$; symbols as in Fig. 2.

in the range of intermolecular distances sampled, Eq. (3) could still be expected to provide an "effective" description of processes which occur on a time scale much slower than molecular rotations, since any such process would sample only the spherical average of the anisotropic potential. Such processes do not, however, include the present scattering experiment. For a thermal rigid rotor at $300 \mathrm{~K}$, representative of the secondary beams used in the present studies (Sec. II), a typical rotation frequency is $\sim 10^{12} \mathrm{~Hz}$ (for $\mathrm{N}_{2}$ ). Under our experimental conditions, the collision interaction time (to traverse $\sim 8 \AA$ ) is $\sim 5 \times 10^{-13} \mathrm{sec}$, permitting the molecule only about half a revolution during the collision.

Interpretations of scattering results for anisotropic interactions have been based for some time on semiclassical calculations. ${ }^{12,13}$ These showed that the effect of anisotropic potential scattering on integral elastic cross sections is primarily to dampen, or quench, the glory undulations. ${ }^{34}$ Similar results were obtained for the supe rnunerary rainbow and rapid quantum oscillatory structure of elastic DCS's. ${ }^{34}$ On this basis, "total" (elastic plus inelastic) DCS's not exhibiting the quenching effect relative to corresponding DCS's from spherically symmetric potentials have usually been interpreted

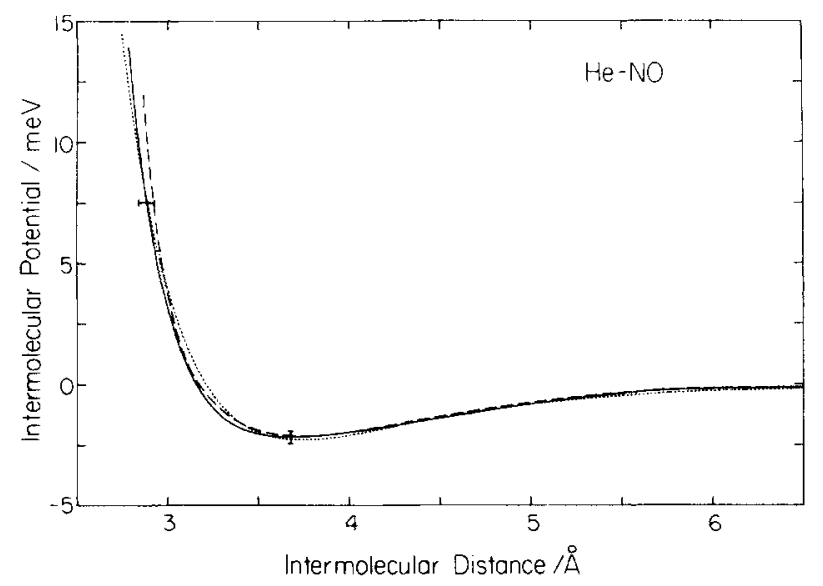

FIG. 5. Spherically symmetric intermolecular potentials for $\mathrm{He}+\mathrm{NO}$; symbols as in Fig. 2. 
as sensitive only to the spherical average of the intermolecular potential. 9 Such a straightforward application of the semiclassical theory for DCS's is however, inappropriate for the highly quantum systems under discussion here. As an example of the differences between semiclassical and quantum scattering, we note that the present DCS's exhibit strong undulations well beyond the classical rainbow angle (at $\sim 4^{\circ}$ for these systems); semiclassical oscillations should not appear on the dark (wide-angle) side of the rainbow, ${ }^{9 c}, 34$ unless the effect of complex trajectories is included. ${ }^{35}$

More recently, quantum mechanical calculations have been performed for scattering from anisotropic potentials. These calculations are extremely involved, and are expensive to perform exactly by close-coupling techniques ${ }^{36}$; "routine" calculations more wieldy for data reduction require the use of various approximation schemes. ${ }^{37}$ One of the most successful of these is the infinite-order sudden approximation (IOSA), ${ }^{14 a}$ which should be accurate when the collision energy is substantially greater than the rotational spacing of the molecular partner. ${ }^{14 b}$ This condition is satisfied in the present case: the most probable rotational levels at $298 \mathrm{~K}$ are separated by $\sim 3 \mathrm{meV}$, whereas the collision energy is $\sim 64 \mathrm{meV}$ (Sec. II). Computationally, the IOSA involves taking cuts along the potential energy surface for various angles $\gamma$ between the molecular axis and the position vector of the approaching He atom with respect to the diatom center of mass. The resulting potentials depend only parametrically on $\gamma$, allowing central-field scatter ing calculations to be performed at each such $\gamma$. The DCS's obtained in this manner are then simply spherically averaged ${ }^{14 a}$ by an eight-point Gauss-Legendre quadrature to yield the IOSA scattering pattern resulting from the assumed anisotropic potential. Although this procedure appears to be tantamount to assuming that the angle $\gamma$ remains fixed during the collision (which would be incorrect, as mentioned at the beginning of this section), it has been shown that the IOSA formulation need assume only that the rotational energy level spacing, and the differences in centrifugal potentials for adjacent orbital angular momentum channels, are small. ${ }^{14 a} \mathrm{We}$ note that the IOSA DCS's calculated in the above fashion are degeneracy averaged over initial, and summed over final, rotational states. This is also true of our experimental measurements, which were performed with a nearly thermal distribution of internal rotational states (Sec. II), and without detection of rotational inelasticities. We may therefore directly compare the IOSA DCS's with the experimental measurements, after suitable coordinate transformations and instrumental averaging effects are included in the calculations (Sec. II).

In fitting the anisotropic MSV potential model of Eqs. (4) and (6) to the DCS data, it was necessary to keep the number of adjustable parameters limited to five. ${ }^{23}$ Consequently, we fix the $\epsilon_{2} / \epsilon_{0}$ ratio, but fit the $r_{q}$ anisotropy parameter of Eq. (6). We predicate this choice on the demonstrated sensitivity of the rapid quantum oscillatory structure on the attractive well position anisotropy, and on its relative lack of sensitivity to the well depth anisotropy. ${ }^{14 b}$ A suitable estimate for the well depth anisot- ropy was taken as $\epsilon_{2} / \epsilon_{0}=-0.2$, mimicking the behavior of an electron-gas calculation for the $\mathrm{He}+\mathrm{CO}$ potential with angles $\gamma=0$ or $\pi$ and $\gamma=\pi / 2 .{ }^{36 b}$ Note that $\epsilon_{2} / \epsilon_{0}<0$ implies that the absolute minimum of the van der Waals potential energy surface is along $\gamma=\pi / 2$. The resulting fitted potentials, whose parameters are given in Table III, are seen to be only weakly anisotropic. For example, for the $\mathrm{He}+\mathrm{NO}$ system, which exhibits the largest absolute value of the anisotropy parameter $r_{q}$, the ratio of $r_{m}$ at $\gamma=0$ to that at $\gamma=\pi / 2$ is only 1.2. Referring to the $\Delta \alpha_{0.95} / \alpha$ columns of Tables II and III, we see that for the $\mathrm{He}+\mathrm{N}_{2}$ system, the anisotropic model does not fit the data any better than do the central-field models having the same number of parameters ( $M^{2}$ SV and SPFD potentials of Table II). The $r_{q}$ parameters obtained from the fits to the $\mathrm{He}+\mathrm{O}_{2}$ and $\mathrm{He}+\mathrm{CO} \mathrm{DCS}$ data indicate that radial anisotropies for these systems are more pronounced than for $\mathrm{He}+\mathrm{N}_{2}$. Despite this, the DCS data are not fit strikingly better by the anisotropic potential model than by the central-field one. In contrast to these three systems, the anisotropic potential fit to the $\mathrm{He}+\mathrm{NO}$ DCS does yield a substantial improvement over any of the central-field fits. In addition, we note that the $r_{a}$ radial anisotropy parameter is more substantial for $\mathrm{He}+\mathrm{NO}$ than for any of the other systems considered in these studies (Table III). Because of the ${ }^{2} \Pi$ electronic symmetry of the NO molecule, $\mathrm{He}+\mathrm{NO}$ collisions at thermal energies involve two potential energy surfaces. ${ }^{30}$ However, since these experiments were performed using randomly oriented NO molecules, these closely-related ${ }^{38}$ potentials may be averaged, yielding a single effective potential having axial symmetry. We note that, for all four of these systems, the $r_{a}$ parameter is negative, implying that the major axis of the fitted elliptical potential is parallel to the molecular axis, as would be expected.

We must regard with some caution however, the relative sizes of the $r_{q}$ anisotropy parameters presented in Table III. ${ }^{39}$ From the $\mathrm{He}+\mathrm{N}_{2}$ fit, it is apparent that the present DCS data can be accurately described, and well fit, by a central-field potential when the $r_{q}$ parameter is smaller in magnitude than $\sim 0.15$. In addition, quenching of the oscillatory structure or other obvious manifestations of anisotropy are not present in our DCS data. Indeed, the experimental DCS's presented in Fig. 1 are quite similar in overall appearance to the $\mathrm{He}+\mathrm{Ar}$ DCS of Paper $\Pi .^{8}$ This is shown in Fig. 6, which displays the latter DCS (also for scattering at a relative collision energy of $64 \mathrm{meV}$ ), for a similar range of scattering angles as used in this study, as well as the present He + NO DCS. Both these DCS's, as well as the $\mathrm{He}+\mathrm{N}_{2}, \mathrm{O}_{2}$, and CO DCS's (Fig. 1), exhibit a vestige of rainbow scattering at $\sim 4^{\circ}$, and all show the highly oscillatory structure at the wider angles that is characteristic of systems with relatively long de Broglie wavelengths and shallow attractive wells. ${ }^{7-9,24}$ In addition, the present scattering results may be fitted reasonably well with potential models that are spherically symmetric (Table II). This is so despite the fact that some of the experimental DCS's and those calculated from the anisotropic potentials [Eq. (6) and Table III], show a slight loss of undulatory amplitude as compared to the best-fit (central- 


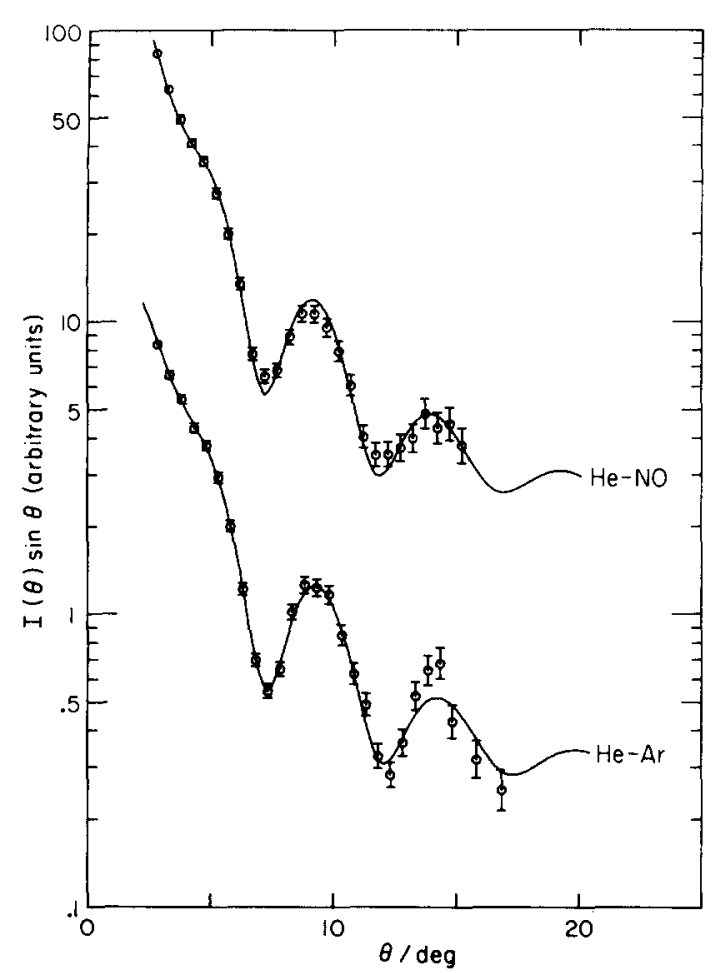

FIG. 6. Laboratory differential cross sections ${ }^{18}$ for out-ofplane scattering of $\mathrm{He}$ by $\mathrm{NO}$ and by $\mathrm{Ar}_{\text {; }}$ the latter are shifted downwards by one decade for clarity of display. The He $+\mathrm{Ar}$ DCS is reproduced from Paper II $^{8}$ for comparison with the $\mathrm{He}+$ NO DCS. Points with error bars are as in Fig. 1. The curves are DCS's calculated from the fitted central-field SPFD potentials. Note the overall similarity between the two cross sections, but compare this to the slight dampening of the rapid quantum oscillatory structure in $\mathrm{He}+\mathrm{NO}$ at $\theta=7^{\circ}, 9^{\circ}$, and $12^{\circ}$, relative to the best-fit central-field calculation; the $\mathrm{He}+\mathrm{Ar}$ DCS shows no analogous effect.

field) calculations (Fig. 1). The potential parameters for the molecular partners are all quite similar to those of the $\mathrm{He}+\mathrm{Ar}$ pair (Paper II). This is quite reasonable, based on the empirical observation that the well depth value $\epsilon$ depends primarily on the polarizability of the least polarizable partner, ${ }^{24}$ which in all these cases is He.

The above similarities between the present slightly anisotropic potentials and the central-field $\mathrm{He}+\mathrm{Ar}$ one suggest that the DCS's presented in this paper may be interpreted with a fair degree of confidence as due primarily to central-field scattering. Similar arguments have been used in the past to justify the centralfield potentials obtained from the analysis of scattering for anisotropic interactions. ${ }^{9,15}$ However, as has recently been suggested theoretically ${ }^{142}$ and confirmed experimentally, ${ }^{11}$ this justification is invalid for highly anisotropic interactions such as $\mathrm{He}+\mathrm{CO}_{2}$. More quantitative evidence is therefore required to justify the central-field potentials' validity for the somewhat less anisotropic systems considered herein and elsewhere. ${ }^{9}$ Evidence of this kind is provided by comparing the spherical averages of the anisotropic potentials with the central-field potentials, as extracted from the present DCS data (Tables $I I$ and $I I$, and Figs. 2-5). We see that the central-field potentials are apparently indistin- guishable from the spherical average of the anisotropic potentials. Thus, for the present systems, which are only slightly anisotropic and which show little evidence for quenching of the rapid quantum oscillatory structure in the DCS (Fig. 6), the central-field analysis may be used to obtain intermolecular potentials which closely approximate the spherical averages of the actual anisotropic interactions.

\section{B. Comparison with other potentials}

We now compare the present spherically symmetric potentials with earlier proposed ones. We do not, however, consider results from bulk property measurements (e.g., virial coefficients, viscosity data, etc). This is because the bulk properties of highly quantum systems are usually sensitive only to a part of the repulsive wall to which the DCS data are insensitive. ${ }^{8}$ All four of the systems studied here have been investigated by measuring arbitrarily-normalized integral scattering cross sections $Q$ as a function of the relative collision velocity $v^{21}$; absolute $Q(v)$ measurements have also been reported for $\mathrm{He}+\mathrm{O}_{2}{ }^{22}$ The arbitrarily-normal ized $Q(v)$ provide data sensitive to the "area" of the attractive well, which is usually expressed as the product $\epsilon r_{m},{ }^{1 b}$ and is dependent upon the model chosen to describe the interaction. The $\epsilon r_{m}$ products for a LJ12-6 model are presented for the $Q(v)$ results in Table IV. To provide an indication of the consistency of our DCS measurements with these results, both of which are sensitive to the attractive well region, we fitted the LJ12-6 model to our scattering data (Sec. III). We list the corresponding $\epsilon r_{m}$ products in the same table, where we see that the DCS values are in reasonable agreement with the parameters obtained from the $Q(v)$ results. We note however, that these values are no more reliable than is the rather crude LJ12-6 model, and are presented here only as a rough estimate of the compatibility of the $Q(v)$ and DCS results. ${ }^{8}$ More reliable values for the $\epsilon r_{m}$ products may be obtained from the more accurate five-parameter potentials, and are presented in Table IV.

Also in Table IV, we compare our fitted "effective" $C_{6}^{\prime}$ dispersion coefficients (Sec. III) with those obtained theoretically. The experimentally determined coefficients for $\mathrm{He}+\mathrm{O}_{2}, \mathrm{CO}$, and $\mathrm{NO}$ are seen to be $\sim 20 \%$ higher than the corresponding calculated ones. ${ }^{29-31}$ This is expected from our neglect of dispersion interaction contributions of higher order than $C_{B}$ (Sec. III). However, the discrepancy for $\mathrm{He}+\mathrm{N}_{2}$ is almost a factor of $2,{ }^{31}$ with our experimental value appearing to be anomalously high with respect to the other experimental values. This difference may be related to our lack of very low angle measurements. We therefore place less confidence in our $\mathrm{He}+\mathrm{N}_{2}$ long-range potential than in our long-range potentials for $\mathrm{He}+\mathrm{O}_{2}, \mathrm{CO}$, and NO. We also compare the present $\mathrm{He}+\mathrm{CO}$ potential to that calculated from the electron-gas formulation. ${ }^{36 b}$ The latter's spherically symmetric component has $r_{m}=3.30 \AA$, $\sigma=2.98 \AA$, and $\epsilon=2.8 \mathrm{meV}$. The theoretical distance parameters appear to be too small by $\sim 0.4 \AA$, while the well is too deep by perhaps $\sim 25 \%$. This is not too surprising, since it is known that the electron-gas method 
TABLE IV. Potential parameters obtained from DCS results, integral cross section measurements, and calculated dispersion coefficients.

\begin{tabular}{|c|c|c|c|c|c|c|c|}
\hline \multirow[b]{2}{*}{ Potential quantity } & \multicolumn{4}{|c|}{ system } & \multirow[b]{2}{*}{ error } & \multirow{2}{*}{$\begin{array}{c}\text { Method of } \\
\text { determination }\end{array}$} & \multirow[b]{2}{*}{ Ref. } \\
\hline & $\mathrm{He}+\mathrm{N}_{2}$ & $\mathrm{He}+\mathrm{O}_{2}$ & $\mathrm{He}+\mathrm{CO}$ & $\mathrm{He}+\mathrm{NO}$ & & & \\
\hline \multirow{4}{*}{$\epsilon r_{m} /(\mathrm{meV} \AA)$} & 8.3 & 9.1 & 8.3 & 8.8 & 0.6 & $Q(v)^{\mathbf{a}}$ & 21 \\
\hline & $\cdots$ & 8.6 & $\cdots$ & $\ldots$ & $\cdots$ & $Q(v)^{\mathbf{a}}$ & 22 \\
\hline & 8.8 & 10.0 & 8.5 & 10.4 & $\cdots$ & $\mathrm{DCS}^{\mathrm{a}}$ & this work \\
\hline & 8.4 & 9.2 & 8.2 & 8.1 & $0.4^{\mathrm{b}}$ & $\mathrm{DCS}^{\mathrm{b}}$ & this work \\
\hline \multirow[t]{3}{*}{$C_{6} /\left(\mathrm{eV} \AA^{6}\right)$} & $\cdots$ & $\cdots$ & 6.7 & $\cdots$ & 0.8 & calculated $^{\mathbf{e}}$ & 29 \\
\hline & $\cdots$ & $\cdots$ & $\cdots$ & 5.8 & 0.5 & calculated $^{c}$ & 30 \\
\hline & 6.1 & 5.6 & $\cdots$ & 6.0 & $\cdots$ & calculated $^{\mathrm{c}}$ & 31 \\
\hline$C_{6}^{\prime} /\left(e V \AA^{6}\right)$ & 11 & 7 & 8 & 7 & 52 & $\operatorname{DCS}^{d}$ & this work \\
\hline
\end{tabular}

a Using the LJ12-6 potential.

Using the central-field $M^{2} S V$ and SPFD potentials, as well as the spherically averaged anisotropic MSV potential. Error reflects the discrepancies among the $\epsilon r_{m}$ products for these three potential forms.

'Spherically symmetric contributions to $C_{6}$.

${ }^{d}$ Using the central-field $M^{2} S V$ and SPFD potentials, and neglecting contributions higher than $C_{6}$ to the dispersion interaction. The error reflects the $\pm 20 \%$ or poorer accuracy with which the "attractive tail" portion of the potential $(r>5 \AA)$ is determined. ${ }^{23}$ (Sec. III).

does not yield very good results for attractive interactions of He-containing systems. ${ }^{5 \mathrm{c}, \mathrm{d}}$ This may also be responsible for the observation that the theoretical anisotropies ${ }^{360}$ for the well region of the $\mathrm{He}+\mathrm{CO}$ potential appear to be somewhat larger than indicated by the $r_{a}$ parameter obtained from the present analysis of DCS scattering results.

Finally, we compare in Fig. 7 the spherically averaged anisotropic potentials of the four scattering systems described in the present paper. As is already evident from Tables II and III, and is more graphically seen in the figure, the $\mathrm{He}+\mathrm{O}_{2}$ potential's attractive minimum occurs at a significantly shorter intermolecular separation than do those of $\mathrm{He}+\mathrm{N}_{2}, \mathrm{CO}$; and $\mathrm{NO}$; the attractive minimum is also somewhat deeper for $\mathrm{He}+\mathrm{O}_{2}$. We also note that the repulsive walls of the systems studied generally have the same steepness (except for $\mathrm{He}+\mathrm{NO}$ ), but that the intermolecular distance at which they occur (as indicated by the value of $\sigma$, for example) appear to be somewhat different. These differences are not correlated with the equilibrium bond lengths of the diatomic molecules $\left(\mathrm{O}_{2}>\mathrm{NO}>\mathrm{CO}>\mathrm{N}_{2}\right)$. In determining the potentials for these systems, it was hoped that some such simple correlation could be found between the electronic or molecular structures of these molecules and their van der Waals interactions with He atoms. Thus, $\mathrm{N}_{2}$ and $\mathrm{CO}$ were naively expected to yield closely related spherically averaged potentials. Similarly, NO (with one unpaired electron) was expected to show some of the characteristics apparently peculiar to $\mathrm{O}_{2}$ (with two unpaired electrons), such as those mentioned above. The lack of any such simple correlations is testimony to the complexity of van der Waals interactions in the weakly repulsive and attractive minimum regions; only the long-range potentials are as yet well understood.

\section{SUMMARY AND CONCLUSIONS}

By measuring the differential cross sections (DCS) for scattering of a room-temperature hypersonic beam of He by crossed beams of $\mathrm{N}_{2}, \mathrm{O}_{2}, \mathrm{CO}$, and NO, we have determined intermolecular potentials describing their van der Waals interactions in the attractive minimum and adjacent regions of the ppotential. ${ }^{23}$ The extraction of these intermolecular potentials were based initially on the central-field assumption, since there was little evidence for quenching of the experimental DCS's, which have been predicted, ${ }^{12-14}$ and observed, ${ }^{11,15}$ for scattering from highly anisotropic potentials.

A more detailed analysis, using a convenient and realistic anisotropic intermolecular potential model, together with the infinite-order sudden approximation, ${ }^{14 a}$ was used to show that anisotropy contributions are appreciable only for $\mathrm{He}+\mathrm{NO}$, being negligible for $\mathrm{He}+\mathrm{N}_{2}$, and small for $\mathrm{He}+\mathrm{O}_{2}$ and $\mathrm{He}+\mathrm{CO}$. The present study provides examples of anisotropic interactions which are too weak to significantly distort the DCS scattering pat-

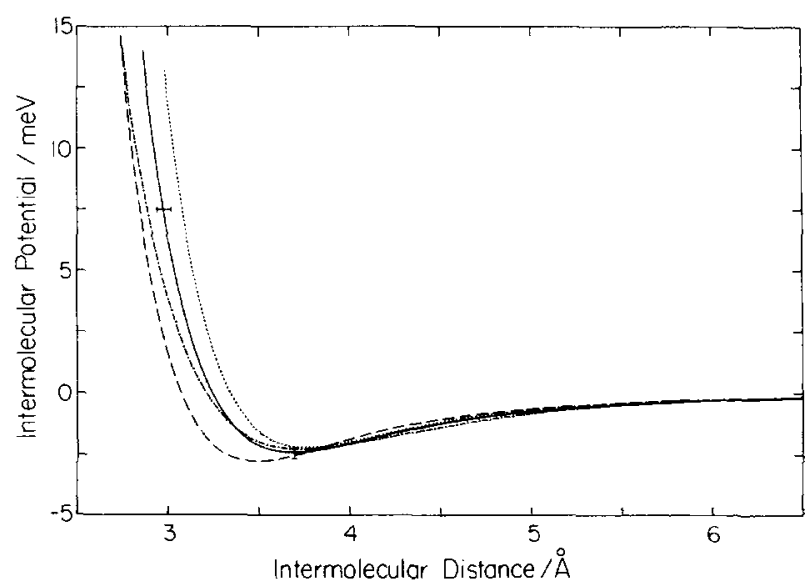

FIG. 7. Spherical averages of the anisotropic MSV potential model of Eqs. (4) and (6), as a function of the intermolecular separation. Solid curve: $\mathrm{He}+\mathrm{N}_{2}$; dashed curve: $\mathrm{He}+\mathrm{O}_{2}$; dotted curve: $\mathrm{He}+\mathrm{CO}$; dash-dotted curve: $\mathrm{He}+\mathrm{NO}$. The error bars on the $\mathrm{He}+\mathrm{N}_{2}$ potential have the same meanings as in Fig. 2. 
terns from those expected from central-field interactions. Indeed, the central-field potentials are indistinguishable, within experimental error, from the spherical averages of the present anisotropic potentials. This finding is in strong contrast to scattering results for more highly anisotropic interactions, such as $\mathrm{He}+\mathrm{CO}_{2} \cdot{ }^{11}$ For weakly anisotropic interactions in highly quantum systems, the central-field assumption thus provides a valid framework for the extraction of intermolecular potentials from thermal scattering data lacking discrimination between elastic and inelastic contributions. Consequently, of course, more detailed experimental information, such as the measurement of inelastic cross sections, will be required for determining anisotropic contributions to the intermolecular potentials for such systems from DCS scattering data. ${ }^{40,41}$

\section{ACKNOWLEDGMENTS}

We wish to thank Dr. Gregory Parker for many useful discussions concerning the infinite-order sudden approximation, and for much help with its use. We also thank Ambassador College for generous use of its computing facilities.

${ }^{1}$ For recent reviews, see (a) U. Buck, Adv. Chem. Phys. 30, 313 (1975); (b) J. P. Toennies, in Physical Chemistry: an Advanced Treatise, Vol. 6A, edited by H. Eyring, D. Henderson, and W. Jost (Academic, New York, 1974), p. 227; (c) G. C. Maitland and E. B. Smith, Chem. Soc. Rev。2, 181 (1973); (d) Ch. Schlier, Ann. Rev. Phys. Chem. 20, 191 (1969).

${ }^{2}$ J. O. Hirschfelder, C. F. Curtiss, and R. B. Bird, Molecular Theory of Bases and Liquids (Wiley, New York, 1954).

${ }^{3}$ G. L. Pollack, Rev. Mod. Phys. 36, 748 (1964).

${ }^{4}$ For examples, see (a) I. Amdur and J. E. Jordan, Adv. Chem. Phys. 10, 29 (1966); (b) J. M. Parson, P. E. Siska, and Y. T. Lee, J. Chem. Phys. 56, 1511 (1972); (c) D. E. Freeman, K. Yoshino, and Y. Tanaka, ibid. 61, 4880 (1974); (d) A. L. J. Burgmans, J. M. Farrar, and Y. T. Lee, J. Chem. Phys. 64, 1345 (1976), and references quoted in these papers.

${ }^{5}$ See (a) A. A. Abrahamson, Phys. Rev. 130, 693 (1963); (b) J. O. Hirschfelder and W. J. Meath, Adv. Chem. Phys. 12, 3 (1967); (c) Y. S. Kim and R. G. Gordon, J. Chem. Phys. 61, 1 (1974); (d) J. S. Cohen and R. T Pack, ibid. 61, 2372 (1974); (e) P. D. Dacre, Chem. Phys. Lett. 50, 147 (1977).

6(a) J. H. Dymond and B. J. Alder, J. Chem. Phys. 51, 309 (1969); (b) R. J. LeRoy, M. L. Klein, and I. J. McGee, Mol. Phys. 28, 587 (1974); (c) R. A. Aziz and H. H. Chen, J. Chem. Phys. 67, 5719 (1977).

${ }^{7}$ K. M. Smith, A. M. Rulis, G. Scoles, R. A. Aziz, and V. Nain, J. Chem. Phys. 67, 152 (1977).

${ }^{8}$ M. Keil, J. T. Slankas, and A. Kuppermann, "Scattering of thermal $\mathrm{He}$ beams by crossed atomic and molecular beams. I. The He-Ar van der Waals potential," J. Chem. Phys. (in press). Hereafter referred to as Paper II.

${ }^{9}$ For examples involving molecular scattering of light systems, see (a) D. H. Winnicur, A. L. Morsound, W. R. Deveraux, L. R. Martin, and A. Kuppermann, J. Chem. Phys. 52, 3299 (1970); (b) J. M. Farrar and Y. T. Lee, ibid. 57, 5492 (1972); (c) A. Kuppermann, R. J. Gordon, and M. J. Coggiola, Faraday Discuss. Chem. Soc. 55, 145 (1973); (d) R. W. Bickes, G. Scoles, and K. M. Smith, Can. J. Phys. 53, 435 (1975); (e) A. M. Rulis and G. Scoles, Chem. Phys. 25, 183 (1977).

${ }^{10}$ (a) R. J. LeRoy, J. S. Carley, and J. E. Grabenstetter,
Faraday Discuss. Chem. Soc. 62, 169 (1977); (b) R. E. Smalley, L. Wharton, and D. H. Levy, J. Chem. Phys. 68, 671 (1978); (c) W. Klemperer, Faraday Discuss. Chem. Soc. 62, 179 (1977); (d) J. Reuss, Adv. Chem. Phys. 30, 389 (1975); (e) R. Shafer and R. G. Gordon, J. Chem。 Phys. 58, 5422 (1973).

${ }^{11} \mathrm{M}$. Keil, G. A. Parker, and A. Kuppermann, "An empirical anisotropic potential for $\mathrm{He}-\mathrm{CO}_{2}$," Chem. Phys. Lett. (in press).

${ }^{12}$ R. E. Olson and R. B. Bernstein, J. Chem. Phys. 49, 162 (1968).

${ }^{13}$ R. J. Cross, ibid. 52, $5703(1970)$.

${ }^{14}$ (a) G. A. Parker and R. T Pack. J. Chem. Phys. 68, 1585 (1978); (b) R. T Pack, Chem. Phys. Lett. 55, 197 (1978).

${ }^{15}$ (a) K. G. Anlauf, R. W. Bickes, and R. B. Bernstein, J. Chem. Phys. 54, 3647 (1971); (b) M. Cavallini, M. G. Dondi, G. Scoles, and U. Valbusa, Chem. Phys. Lett. 10, 23 (1971); (c) F. P. Tully and Y. T. Lee, J. Chem. Phys. 57, 866 (1972).

${ }^{16} \mathrm{~J}$. B. Anderson and J. B. Fenn, Phys. Fluids 8, 780 (1965).

${ }^{17}$ E. F. Greene, M. H. Lau, and J. Ross, J. Chem. Phys. 50, 3122 (1969). See also Paper II.

${ }^{18}$ It is convenient to refer to $I(\theta)$ as a laboratory DCS in arbitrary units. However, $I(\theta)$ is really a signal intensity. To transform it into such a laboratory DCS requires multiplication by the laboratory velocity of the scattered beam (which is a function of $\theta$ ), since the mass spectrometer used in our measurements is a number density detector. See Ref, 8 for details.

${ }^{19}$ D. W. Marquardt, J. Soc. Indust. Appl. Math. 11, 431 (1963).

${ }^{20}$ N. R. Draper and H. Smith, Applied Regression Analysis (Wiley, New York, 1966), Chaps. 2 and 9.

${ }^{21}$ H. P. Butz, R. Feltgen, H. Pauly, and H. Vehmeyer, Z. Phys. 247, 70 (1971).

${ }^{22}$ E. Luzzatti, F. Pirani, and F. Vecchiocattivi, Mol. Phys. 34, 1279 (1977)

${ }^{23} \mathrm{M}$. Keil and A. Kuppermann, "Scattering of thermal He beams by crossed atomic and molecular beams. I. Sensitivity of the elastic differential cross section to the interatomic potential," J. Chem. Phys. 69, 3917 (1978).

${ }^{24}$ C. H. Chen, P. E. Siska, and Y. T. Lee, J. Chem. Phys. 59, 601 (1973).

${ }^{25}$ J. M. Parson and Y. T. Lee, Entropie 42, 146 (1971). Our MSV potential differs in the choice of the spline point ${ }^{23}$ (see text).

${ }^{26} \mathrm{Our} \mathrm{M}^{2} \mathrm{SV}$ potential is somewhat similar to the MMSV part of the ESMMSV potential form proposed in Ref. 4(d). However, we join the two Morse functions at $\sigma$, rather than at $r_{m}{ }^{8}$

${ }^{27}$ G. Simons, R. G. Parr, and J. M. Finlan, J. Chem. Phys. 59, 3239 (1973).

${ }^{28}$ R. W. Bickes and R. B. Bernstein, Chem. Phys. Lett. 26, 457 (1974). We use the long-range behavior to fix two of the SPFD coefficients by smoothness conditions, rather than the reverse.

${ }^{29}$ G. A. Parker and R. T Pack, J. Chem. Phys. 64, 2010 (1976).

${ }^{30}$ G. C. Nielson, G. A. Parker, and R. T Pack, J. Chem. Phys. 64, 2055 (1976).

${ }^{31}$ D. J. Margoliash and W. J. Meath, J. Chem. Phys. 68, 1426 (1978).

${ }^{32}$ B. Rosenblum, A. H. Nethercot, and C. H. Townes, Phys. Rev, 109, 400 (1958); C. A. Burrus, J. Chem. Phys. 28, 427 (1958).

${ }^{33}$ C. A. Burrus and J. D. Graybeal, Phys. Rev. 109, 1553 (1958).

${ }^{34}$ R. B. Bernstein, Adv. Chem. Phys. 10, 75 (1966).

${ }^{35}$ R. J. Gordon, J. Chem. Phys. 63, 3109 (1975).

${ }^{36}$ See (a) W. A. Lester, Methods Comput. Phys, 10, 211 (1971), and references quoted therein. For more recent results, see for example, (b) S. Green and P. Thaddeus, Astrophys. 
J. 205, 766 (1976); (c) L. Monchick, J. Chem. Phys. 67, 4626 (1977); (d) M. H. Alexander, ibid. 67, 2703 (1977).

${ }^{37}$ See for example, L. Monchick and S. Green, J. Chem. Phys. 66, 3085 (1977).

${ }^{38}$ The spin-orbit splitting between the ${ }^{2} \Pi_{1 / 2}$ and ${ }^{2} \Pi_{3 / 2}$ states of NO is $15 \mathrm{meV}$, G. Herzberg, Spectra of Diatomic Molecules (van Nostrand Reinhold, New York, 1950), p. 558. For the $\mathrm{Ar}+\mathrm{NO}$ system, the electron-gas calculations of G. C. Nielson, G. A. Parker, and R. T Pack, J. Chem. Phys. 66, 1396 (1977) indicate that this spitting remains small for all geometries of importance for thermal-energy scattering. A similar situation is expected to prevail for the $\mathrm{He}+$ NO sys tem.
${ }^{39}$ Within the elliptical parameterization for the anisotropic potential [Eq. (6)], the $r_{\alpha}$ parameter is statistically independent of the other MSV parameters. This indicates the possibility of obtaining meaningful descriptions of the radial anisotropies. See also Refs. 10(a) and 11.

${ }^{40} \mathrm{U}$. Buck, F. Huisken, J. Schleusener, and H. Pauly, Phys. Rev. Lett. 38, 680 (1977); W. R. Gentry and C. F. Giese, J. Chem. Phys, 67, 5389 (1977).

${ }^{41} \mathrm{~J}$. M. Farrar, J. M. Parson, and Y. T. Lee, Proceedings of the 4th International Symposium on Molecular Beams (Cannes, France, 1973), p. 215; H. J. Loesch, Chem. Phys. Lett. 18, $431(1976)$; N. C. Blais, J. B. Cross, and G. H. Kwei, J. Chem. Phys. 66, 2488 (1977). 